Review

\title{
Concise Review: Comparison of Culture Membranes Used for Tissue Engineered Conjunctival Epithelial Equivalents
}

\author{
Jon Roger Eidet ${ }^{1, *}$, Darlene A. Dartt ${ }^{2}$ and Tor Paaske Utheim ${ }^{3,4,5}$ \\ 1 Department of Ophthalmology, Oslo University Hospital, Oslo 0424, Norway \\ 2 Schepens Eye Research Institute, Massachusetts Eye and Ear/Harvard Medical School, \\ Boston, MA 02114, USA; E-Mail: Darlene_Dartt@meei.harvard.edu \\ 3 Department of Medical Biochemistry, Oslo University Hospital, Oslo 0424, Norway; \\ E-Mail: utheim2@gmail.com \\ 4 Department of Oral Biology, University of Oslo, Oslo 0316, Norway \\ 5 Department of Ophthalmology, Vestre Viken Hospital Trust, Drammen 3004, Norway \\ * Author to whom correspondence should be addressed; E-Mail: j.r.eidet@gmail.com; \\ Tel.: +47-2211-8545; Fax: +47-2211-9989.
}

Academic Editor: Dimitrios Karamichos

Received: 25 October 2015 / Accepted: 7 December 2015 / Published: 11 December 2015

\begin{abstract}
The conjunctival epithelium plays an important role in ensuring the optical clarity of the cornea by providing lubrication to maintain a smooth, refractive surface, by producing mucins critical for tear film stability and by protecting against mechanical stress and infectious agents. A large number of disorders can lead to scarring of the conjunctiva through chronic conjunctival inflammation. For controlling complications of conjunctival scarring, surgery can be considered. Surgical treatment of symblepharon includes removal of the scar tissue to reestablish the deep fornix. The surgical defect is then covered by the application of a tissue substitute. One obvious limiting factor when using autografts is the size of the defect to be covered, as the amount of healthy conjunctiva is scarce. These limitations have led scientists to develop tissue engineered conjunctival equivalents. A tissue engineered conjunctival epithelial equivalent needs to be easily manipulated surgically, not cause an inflammatory reaction and be biocompatible. This review summarizes the various substrates and membranes that have been used to culture conjunctival epithelial cells during the last three decades. Future avenues for developing tissue engineered conjunctiva are discussed.
\end{abstract}


Keywords: conjunctiva; epithelium; biomaterials; membranes; culture; tissue engineering; goblet cells; ocular surface; transplantation

\section{Conjunctiva}

Conjunctival epithelium is non-keratinized and is at least two cell layers thick [1]. The number of cell layers depends on the degree of conjunctival stretching [2]. The conjunctival epithelium consists of two phenotypically distinct cell types, stratified squamous non-goblet cells (90\%-95\%) and goblet cells (5\%-10\%) (Figure 1), in addition to occasional lymphocytes [3] and melanocytes. The conjunctival epithelium plays an important role in ensuring the optical clarity of the cornea by providing lubrication to maintain a smooth, refractive surface, and by producing mucins critical for tear film stability [4]. The conjunctiva also protects the eye against mechanical stress and infectious agents. It, furthermore, contributes water and electrolytes to the tear fluid [5]. The squamous cells produce cell membrane-tethered mucins, while the goblet cells secrete the gel-forming mucins, both of which helps to maintain a protective tear film. The superficial surface of the squamous cells are covered by the membrane-tethered mucins mucin-1 (MUC1), mucin-4 (MUC4) and mucin-16 (MUC16) [6], which are essential for tear stability and make up the glycocalyx [6].
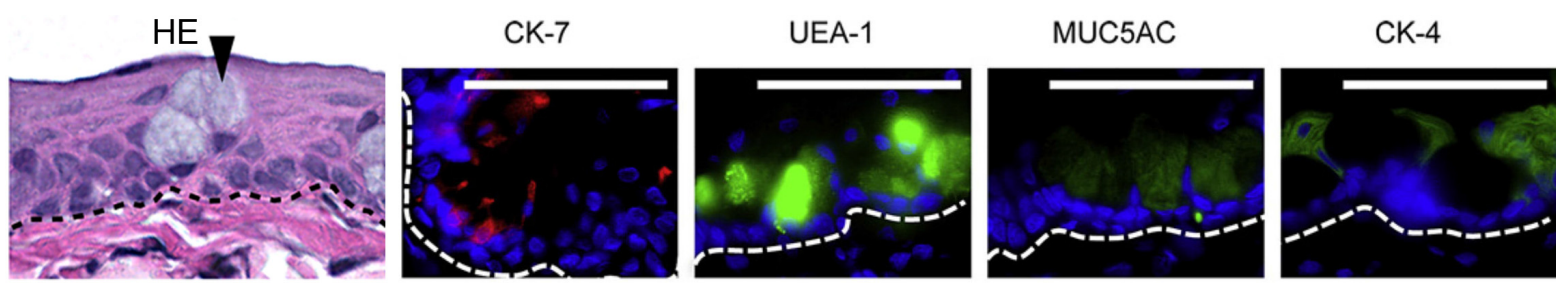

Figure 1. Photomicrographs show hematoxylin and eosin (HE) and immunofluorescently stained sections of rat conjunctiva. The black arrowhead in the HE photomicrograph indicates mucin granules of goblet cells. The black dotted line indicates the basal membrane, which overlies loose vascularized conjunctival forniceal connective tissue. Original magnification of the HE photomicrograph: $\times 630$. Immunofluorescence photomicrographs of forniceal conjunctival sections show conjunctival epithelial cell markers, which include the goblet cell markers anti-cytokeratin 7 (Ck7), Ulex europaeus agglutinin 1 (UEA-1) lectin and anti-mucin 5AC (MUC5AC), as well as the marker for stratified squamous non-goblet cells anti-cytokeratin $4(\mathrm{Ck} 4)$. Nuclei were stained with DAPI (blue). Ck7 is expressed in the goblet cell body, whereas UEA-1 and MUC5AC stain the goblet cell mucin-contents. $\mathrm{Ck} 4$ is only detected in squamous cells between goblet cell clusters. The basal membrane is indicated by the white dotted line. Scale bars: $100 \mu \mathrm{m}$. Adapted from Fostad et al. 2012 [7].

The gel-forming mucin-5AC (MUC5AC) and mucin-2 (MUC2) are secreted by goblet cells into the aqueous layer of the tear film [8,9] (Figure 2). The squamous conjunctival cells also contribute to the hydration of the ocular surface through ion transport across the apical cell membrane with accompanying osmotic water transfer [5]. Goblet cells contain mucin-granules and have traditionally been identified through their secretory product using markers, including the ulex europaeus agglutinin-1 (UEA-1) lectin, 
anti-mucin-5AC (MUC5AC) and anti-AM3 antibodies, and periodic acid-Schiff (PAS) reagent that target the goblet cell gel-forming mucins [10]. In addition to cytokeratin 4 (Ck4) (Figure 1), squamous conjunctival epithelial cells can be identified by $\mathrm{Ck} 13$, a binding pair of $\mathrm{Ck} 4$ [11].

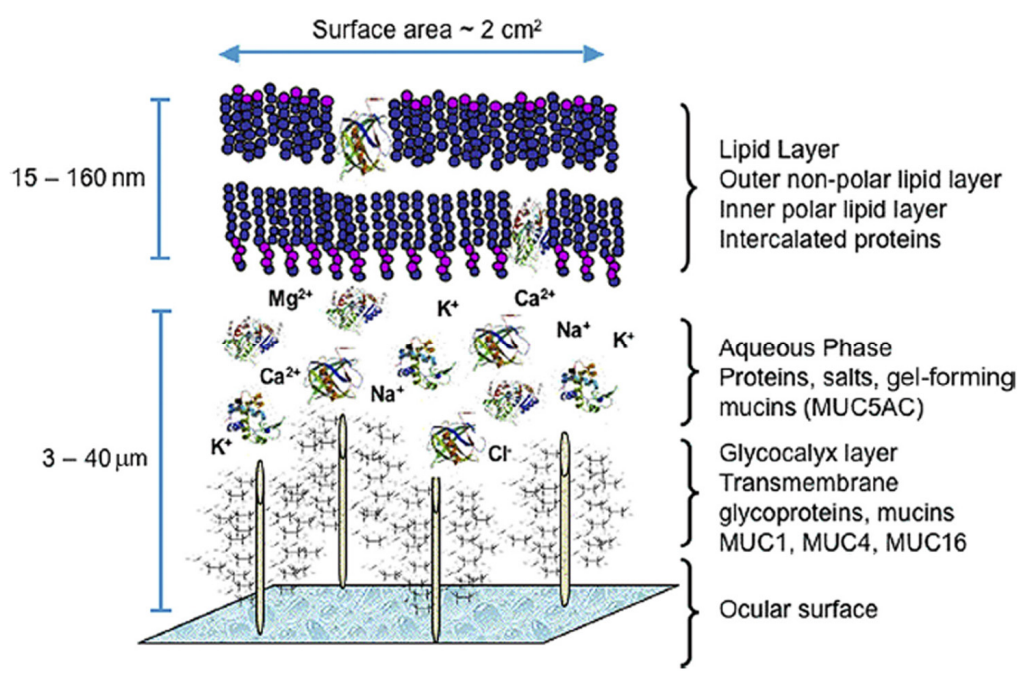

Figure 2. Model of the human tear film. Adapted from Nichols et al. 2001 [12].

\section{Conjunctival Stem Cells}

Conjunctival stem cells continuously regenerate the conjunctiva by giving rise to both stratified squamous non-goblet and goblet cells [13], thereby maintaining a healthy tear film [14]. Disorders that damage these stem cells cause varying extent of keratinization, which disrupts the protective tear film and ultimately leads to limbal stem cell deficiency (LSCD) and visual impairment or blindness. The location of the conjunctival epithelial stem cells has been investigated in several studies on mouse [15-17], rat [18,19], rabbit [20,21] and human [22-24] tissue, yet no real consensus has been reached. The conjunctival stem cells have been suggested to reside in the limbus [18], bulbar conjunctiva [15,22,23], medial canthal [24], forniceal conjunctiva [16,17,20,22,24,25], palpebral conjunctiva [19] and mucocutaneous junction [18,21]. Although the conjunctival stem cells may not solely be located to one single region, their relative number generally appears to be highest in the fornix [26].

Stem cells are surrounded and influenced by a three-dimensional microenvironment known as a niche [27]. The niche comprises of numerous components, including stromal cells, soluble factors, extracellular matrix (ECM), mechanical/spatial cues and signaling molecules that dictates stem cell function [28]. The limbal stem cell niche has been reported to contain specific ECM proteins. Moreover, the specific composition of the ECM shows topographical variations throughout the ocular surface [29]. Thus, the specific composition of the ECM in the substrate may affect the preservation of conjunctival stem cells in culture.

\section{Conjunctival Scarring Diseases}

A large number of disorders can lead to scarring of the conjunctiva through chronic conjunctival inflammation. Scarring varies in severity and can be self-limited, such as in chemical/thermal burns and infectious diseases due to adeno- and herpes viruses, or progressive, as in cicatrizing conjunctivitis, which consists of several diseases including ocular cicatricial pemphigoid, Stevens-Johnson syndrome, 
atopic keratoconjunctivitis and Sjögren's syndrome [30]. Cicatrizing conjunctivitis is rare, and in total these disorders have an incidence of 1.2 in 1 million in the United Kingdom [30]. Treatment depends on the disease etiology and severity, but can include various anti-inflammatory, immunomodulatory and immunosuppressive drugs [31].

Surgical treatment of symblepharon includes removal of the scar tissue to reestablish the deep fornix [32]. The surgical defect is then covered with a tissue substitute to prevent re-obliteration. These include mechanical [33], physical [34] or chemical [35] approaches and the grafting of conjunctival or mucous membranes [32]. Surgical techniques for restoration of a diseased conjunctiva have utilized different conjunctival substitutes, including conjunctival autografts [36]. An obvious limiting factor when using autografts is the size of the defect to be covered, as the amount of healthy conjunctiva is limited. These drawbacks have led scientists to develop tissue engineered conjunctival equivalents.

\section{Tissue Engineered Conjunctival Equivalents}

A tissue engineered conjunctival epithelial equivalent needs to be easily manipulated surgically, not cause an inflammatory reaction, be biocompatible and contain a mix of stratified squamous cells, goblet cells and undifferentiated cells. Unlike tissue engineered corneal equivalents, conjunctival equivalents do not need to be transparent, which increases the range of suitable culture membranes.

In addition to conjunctival epithelial cells (CEC) cultured on amniotic membrane (AM) [4], there is likely a wide range of cell types that can be used for developing a tissue engineered conjunctival equivalent. This assumption is based on multiple studies demonstrating successful restoration of the cornea with cultured non-limbal cells. Tissue engineered corneal equivalents share many of the same prerequisites as conjunctival equivalents, e.g., with regard to barrier function and tear film support. Besides limbal stem cells, corneal equivalents have been developed from oral mucosal epithelial cells [37,38], embryonic stem cells (ESC) [39], bone-marrow-derived mesenchymal stem cells (MSC) [40], immature dental pulp stem cells [41], hair follicle-derived stem cells [42] and umbilical cord lining stem cells [43]. For conjunctival reconstruction, epidermal keratinocytes have been cultured on AM and transplanted to restore the conjunctiva in rhesus monkeys [44]. Although the conjunctival stratified squamous cell markers MUC4 and Ck4 were present in the transplant, goblet cells were absent. In a recent study, a tissue engineered conjunctival equivalent was developed from cultured AM epithelial cells [45]. The conjunctival equivalent contained PAS-positive cells, indicative of goblet cells, and successfully restored the conjunctiva in a rabbit model. Transplants containing goblet cells could also be developed from nasal mucosa, which harbors goblet cells [46]. Thus, there are multiple possible cell sources for developing conjunctival equivalents, though no comparative studies have defined the optimal choice of donor cells.

A number of different substrates and membranes have been attempted for tissue engineering conjunctival epithelial equivalents. These can be categorized into: (1) biological membranes; (2) extracellular matrix protein-containing membranes; and (3) synthetic polymer membranes.

\subsection{Biological Membranes}

Seventy-six years after it was first used in ophthalmology, AM, which constitutes the innermost layer of the fetal membranes, has a prominent role in ocular surface reconstruction [47]. AM is particularly 
suited for clinical use as it supports epithelialization [48], reduces scaring [49], suppresses the immune response [50], reduces pain, and decreases inflammation [51]. Prior to AM transplantation (AMT), the AM is cryopreserved, which kills all the AM cells [52]. Hence, AM grafts function primarily as a matrix and not by virtue of transplanted functional cells. The membranes have most commonly been cryopreserved in a basal cell medium at $-80{ }^{\circ} \mathrm{C}$ [53], but a technique for freeze-drying the $\mathrm{AM}$ has also been developed [54]. Freeze-dried AM can be sterilized by gamma-irradiation [54], however, AM treated this way may release a less amount of growth factors than conventionally cryopreserved membranes [55]. In addition, the AM can be sterilized with per-acetic acid/ethanol and air-dried [56]. The latter technique is, on the other hand, reported to yield inferior results compared to cryopreserved AM with respect to rate of cell outgrowth, release of wound-healing factors, and preservation of the AM basement membrane (BM) [57]. In patients with chronic inflammation there is a tendency for recurrent shrinkage and symblepharon formation after restoring the ocular surface with AM [58]. The success of transplanting AM is therefore dependent on the underlying disease [4].

Twelve studies have described culture of CEC on AM, of which eight used denuded AM (dAM) (Table 1). Meller et al. first reported the use of dAM for cell culture of CEC since they noticed that the devitalized AM epithelium inhibited adhesion and growth of the CEC [59]. All later studies using intact AM have utilized explant culture.

Eight out of ten studies confirmed the presence of goblet cells on AM (detected either by their mucin content or by $\mathrm{Ck} 7$ ), irrespective of whether dAM or intact AM had been used [59]. Data on actual percentages of goblet cells in CEC cultures on AM are sparse, although one study reported that between $25 \%$ and $75 \%$ of the cells were MUC5AC positive [60]. Although Ck7 positive goblet cells have been demonstrated under serum-free conditions, addition of 10\% FBS improved the preservation of goblet cells [61]. This is in line with a study showing that FBS promotes expression of conjunctival epithelial cytokeratins due to the effect of vitamin A [62]. Development of mucin-containing goblet cells have also been achieved on AM independent of feeder cells, air-lifting or high calcium [60]. Thus, AM generally promotes goblet cell development.

Stratified CEC were obtained in all studies using AM, except one [63]. Culture techniques to induce stratification include the use of explants, air-lifting, feeder layer, and high calcium. Air-lifting promotes cell polarity by increasing the number of microvilli, tight junctions, and hemidesmosomes in CEC cultures [59]. The molecular mechanisms involved in air-lifting include the p38 mitogen-activated protein kinase and Wnt signaling pathways [64]. Stratification was achieved when including a feeder layer [50], air-lifting [59] and/or high calcium [65] in cell cultures.

Stratified CEC cultures were also generated on cadaveric acellular dermis (AlloDerm) coated with collagen type 4 (COL4) [65]. The latter study employed a serum-free culture protocol without feeder cells. Goblet cells, however, were not reported. Hence, except for the latter study on acellular dermis, culture of CEC on biological membranes generally promotes stratified cultures with goblet cells. 
Table 1. Conjunctival Epithelial cells cultured on biological membranes.

\begin{tabular}{|c|c|c|c|c|c|c|c|c|c|c|c|c|}
\hline $\begin{array}{l}\text { Substrate } \\
\text { (s) }\end{array}$ & $\begin{array}{c}\text { Cell } \\
\text { Species }\end{array}$ & $\begin{array}{c}\text { Explant/Suspension } \\
\text { Culture }\end{array}$ & $\begin{array}{c}\text { Culture } \\
\text { Time } \\
\text { (Days) }\end{array}$ & $\begin{array}{c}\text { Feeder } \\
\text { Cells }\end{array}$ & $\begin{array}{c}\text { Air- } \\
\text { Lifting }\end{array}$ & $\begin{array}{c}\text { High } \\
\text { Calcium }\end{array}$ & $\begin{array}{c}\text { Basal } \\
\text { Medium }\end{array}$ & Serum & $\begin{array}{c}\text { Conjunctival } \\
\text { Donor Site }\end{array}$ & Goblet Cells & Comment & Authors \\
\hline $\mathrm{AM}$ & Human & Explant & 21 & - & - & - & CNT50 & FBS/AS & - & $\begin{array}{l}\text { Yes (with } \\
\text { both serum } \\
\text { type) }\end{array}$ & & $\begin{array}{l}\text { Rivas et al. } \\
2014\end{array}$ \\
\hline $\mathrm{AM}$ & Human & Explant & 14 & No & No & No & DMEM:F12 & $5 \% \mathrm{FBS}$ & Fornix/bulbus & $\begin{array}{c}\text { Yes }(<25 \% \text { to } \\
75 \% \\
\text { MUC5AC+) }\end{array}$ & $\begin{array}{l}\text { Stratified } \\
\text { culture }\end{array}$ & $\begin{array}{l}\text { Eidet et al. } \\
2014\end{array}$ \\
\hline $\mathrm{AM}$ & Human & Explant & 12 & No & Yes & No & DMEM:F12 & $5 \% \mathrm{FBS}$ & Bulbus & $\begin{array}{c}\text { Yes } \\
\text { (MUC5AC+, } \\
\text { fever than in } \\
\text { native } \\
\text { conjunctiva) }\end{array}$ & $\begin{array}{l}\text { Stratified } \\
\text { culture }\end{array}$ & $\begin{array}{c}\text { Tan et al. } \\
2014\end{array}$ \\
\hline $\mathrm{AM}$ & Rabbit & Explant & $8-15$ & $3 \mathrm{~T} 3 / \mathrm{No}$ & Yes & - & - & - & Fornix & $\begin{array}{c}\text { No } \\
\text { MUC5AC- }\end{array}$ & $\begin{array}{c}\text { Stratified } \\
\text { culture } \\
(\mathrm{Ck} 3+/ \mathrm{Ck} 12-)\end{array}$ & $\begin{array}{c}\text { Cho et al. } \\
2014\end{array}$ \\
\hline $\mathrm{dAM}$ & Human & Explant & $9-11$ & $3 \mathrm{~T} 3$ & Yes & - & DMEM:F12 & $\begin{array}{l}10 \% \\
\text { FBS }\end{array}$ & - & $\begin{array}{c}\text { Yes }(\mathrm{PAS}+\text {, } \\
\text { increased by } \\
\gamma \mathrm{SI})\end{array}$ & $\begin{array}{l}\text { Stratified } \\
\text { culture }\end{array}$ & $\begin{array}{c}\text { Tian et al. } \\
2014\end{array}$ \\
\hline $\mathrm{dAM}$ & Human & Explant & - & No & No & - & DMEM:F12 & FBS & Fornix & Yes (PAS+) & $\begin{array}{l}\text { Stratified } \\
\text { culture }\end{array}$ & $\begin{array}{l}\text { Silber et al. } \\
2014\end{array}$ \\
\hline $\mathrm{dAM}$ & Human & Suspension & 5 & No & No & - & $\begin{array}{l}\text { KM (serum } \\
\text { free or } \\
\text { DMEM:F12) }\end{array}$ & $\begin{array}{l}0 \%- \\
20 \% \\
\text { FBS }\end{array}$ & Palpebra & $\begin{array}{c}\text { Yes }(100 \% \\
\text { Ck7+; best } \\
\text { preserved by } \\
10 \% \mathrm{FBS})\end{array}$ & & $\begin{array}{l}\text { Martinez- } \\
\text { Osorio } \\
\text { et al. } 2009\end{array}$ \\
\hline
\end{tabular}


Table 1. Cont.

\begin{tabular}{|c|c|c|c|c|c|c|c|c|c|c|c|c|}
\hline $\begin{array}{l}\text { Substrate } \\
\text { (s) }\end{array}$ & $\begin{array}{c}\text { Cell } \\
\text { Species }\end{array}$ & $\begin{array}{c}\text { Explant/Suspension } \\
\text { Culture }\end{array}$ & $\begin{array}{c}\text { Culture } \\
\text { Time } \\
\text { (Days) }\end{array}$ & $\begin{array}{c}\text { Feeder } \\
\text { Cells }\end{array}$ & $\begin{array}{c}\text { Air- } \\
\text { Lifting }\end{array}$ & $\begin{array}{c}\text { High } \\
\text { Calcium }\end{array}$ & $\begin{array}{c}\text { Basal } \\
\text { Medium }\end{array}$ & Serum & $\begin{array}{c}\text { Conjunctival } \\
\text { Donor Site }\end{array}$ & Goblet Cells & Comment & Authors \\
\hline $\mathrm{dAM}$ & Human & Suspension & 21 & $3 \mathrm{~T} 3$ & Yes & Yes & $\begin{array}{l}\text { KM (serum } \\
\text { free or } \\
\text { DMEM:F12) }\end{array}$ & FBS & - & $\begin{array}{c}\text { No } \\
\text { (MUC5AC-) }\end{array}$ & $\begin{array}{l}\text { Stratified } \\
\text { culture }\end{array}$ & $\begin{array}{l}\text { Tanioka } \\
\text { et al. } 2006\end{array}$ \\
\hline $\mathrm{dAM}$ & Human & Explant & 14 & No & - & Yes & DMEM:F12 & $\mathrm{FBS} / \mathrm{HS}$ & Bulbus & - & $\begin{array}{l}\text { Stratified } \\
\text { culture }\end{array}$ & $\begin{array}{l}\text { Ang et al. } \\
2005\end{array}$ \\
\hline $\mathrm{dAM}$ & Human & Explant & $12-22$ & No & Yes/No & Yes/No & $\begin{array}{c}\text { KGM or } \\
\text { DMEM/F12 }\end{array}$ & $\begin{array}{l}0 \text { or } \\
10 \% \\
\text { FBS }\end{array}$ & Bulbus & $\begin{array}{c}\text { Yes } \\
\text { (MUC5AC } \\
\text { detected by } \\
\text { PCR in all } \\
\text { groups) }\end{array}$ & $\begin{array}{l}\text { Stratified } \\
\text { culture }\end{array}$ & $\begin{array}{l}\text { Ang et al. } \\
2004\end{array}$ \\
\hline $\mathrm{dAM}$ & Human & Explant & $11-15$ & No & No & - & KGM:F12 & $\begin{array}{l}10 \% \\
\text { FBS }\end{array}$ & Bulbus & - & $\begin{array}{l}\text { Monolayer } \\
\text { culture }\end{array}$ & $\begin{array}{l}\text { Sangwan } \\
\text { et al. } 2003\end{array}$ \\
\hline $\mathrm{dAM}$ & Rabbit & Suspension & $<28$ & $\mathrm{RCF}$ & Yes/No & No & DMEM:F12 & $5 \% \mathrm{FBS}$ & - & $\begin{array}{c}\text { Yes (scattered } \\
\text { MUC5AC+ } \\
\text { cells } \\
\text { with/without } \\
\text { AL and RCF) }\end{array}$ & $\begin{array}{c}\text { Stratified } \\
\text { culture } \\
\text { (increased in } \\
\text { AL) }\end{array}$ & $\begin{array}{c}\text { Meller } \\
\text { et al. } 1999\end{array}$ \\
\hline $\begin{array}{l}\text { AlloDerm } \\
\text { coated } \\
\text { with } \\
\text { COL4 }\end{array}$ & Human & Suspension & 18 & No & Yes & Yes & MCDB 153 & No & - & - & $\begin{array}{l}\text { Stratified } \\
\text { culture }\end{array}$ & $\begin{array}{l}\text { Yoshizawa } \\
\text { et al. } 2004\end{array}$ \\
\hline
\end{tabular}

$\mathrm{AL}=$ air-lifting; $\mathrm{AM}=$ human amniotic membrane; $(-)=$ not reported; AS = autologous serum; DMEM = Dulbecco's Modified Eagle's Medium; 3T3 = 3T3 feeder cells; $\gamma \mathrm{SI}=\gamma$-secretase inhibitor; $\mathrm{dAM}=$ denuded $\mathrm{AM} ; \mathrm{KM}=$ keratinocyte medium; HS = human serum; $\mathrm{KGM}=$ keratinocyte growth medium; RCF $=$ rabbit conjunctival fibroblasts; COL4 = collagen type 4; MUC5AC = mucin 5AC; Ck7 = cytokeratin 7. 


\subsection{Extracellular Matrix Protein-Containing Membranes}

The conjunctival BM is a thin connective tissue membrane, which is composed of collagen type IV (collagen $\alpha 1$ and $\alpha 2$ chains), laminin ( $\alpha 5, \beta 2$ and $\gamma 1$ chains), nidogen- 1 and -2 and thrombospondin-4 [29]. It is therefore reasonable to assume that a tissue engineered CEC equivalent would benefit from being surfaced by ECM proteins. Nineteen studies have described the culture of CEC on various ECM proteins (Table 2). Collagen type 1 (COL1) was most commonly used, either in the form of a coating [66], a gel [67] or as a compressed gel [68]. The latter two forms offer the mechanical strength to transfer the cultured cells to the surgical site. In addition, fibronectin (FN), laminin (LN), Matrigel, elastin-like polymer (ELP), gelatin-chitosan and poly-1-lysine (PLL) were tried [61,66,69-77].

Goblet cells were seen when CEC were grown inside a collagen gel [78], but not always when grown as a monolayer on top of the collagen gel [78]. Compared to Matrigel, CEC grown on COL1 expressed more MUC5AC RNA than Matrigel cultures [76]. Five percent PAS positive goblet cells were detected when culturing CEC on top of a COL1:COL3 mix in serum-free medium [71]. The latter study also achieved stratification. When cultured without feeder cells, air-lifting or high calcium, the CEC formed monolayer cultures on COL1 [66]. Stratified cultures were achieved with the addition of feeder cells [67], air-lifting [67], or high calcium [76].

Matrigel is composed of LN, COL4, heparan sulfate proteoglycans, entactin, transforming growth factor (TGF), and basic fibroblast growth factor (bFGF) [71]. Cultured CEC generally form aggregates on Matrigel rather than continuous cell sheets $[66,76]$. In one study the aggregates contained PAS positive goblet cells [66].

Use of fibronectin, either alone or in a mix with COL1, was reported in four studies [69-72]. The CEC formed monolayer cultures [70], but the presence of goblet cells were not reported. Elastin-like polymer has been used to grow Ck7 positive cells of the cell line IOBA-NHC [79]. Gelatin-chitosan yielded stratified cultures with $\mathrm{Ck} 4$ positive squamous cells when using explant culture [77]. Of all the ECM protein substrates, collagen gels and compressed collagen appear the most useful for conjunctival tissue engineering due to their mechanical properties and potential promotion of goblet cell formation. 
Table 2. Conjunctival epithelial cells cultured on extracellular matrix protein-containing membranes.

\begin{tabular}{|c|c|c|c|c|c|c|c|c|c|c|c|c|c|}
\hline Substrate (s) & $\begin{array}{c}\text { Cell } \\
\text { Species }\end{array}$ & $\begin{array}{c}\text { Normal } \\
\text { Cells/Cells } \\
\text { Line } \\
\end{array}$ & $\begin{array}{c}\text { Explant/ } \\
\text { Suspension } \\
\text { Culture } \\
\end{array}$ & $\begin{array}{c}\text { Culture } \\
\text { Time } \\
\text { (Days) } \\
\end{array}$ & $\begin{array}{c}\text { Feeder } \\
\text { Cells }\end{array}$ & $\begin{array}{c}\text { Air- } \\
\text { Lifting }\end{array}$ & $\begin{array}{c}\text { High } \\
\text { Calcium }\end{array}$ & $\begin{array}{c}\text { Basal } \\
\text { Medium }\end{array}$ & Serum & $\begin{array}{c}\text { Conjunctival } \\
\text { Donor Site }\end{array}$ & Goblet Cells & Comm ent & Authors \\
\hline BSA:COL mix & Rabbit & Normal & Suspension & - & No & No & No & $\begin{array}{l}\text { PC-1 (serum } \\
\text { free) }\end{array}$ & No & - & - & & $\begin{array}{c}\text { Scholz et al. } \\
2002\end{array}$ \\
\hline $\mathrm{COL}$ & Rabbit & Normal & Suspension & 6 & No & Yes/No & No & $\begin{array}{l}\text { PC-1 (serum } \\
\text { free) or } \\
\text { DMEM:F12 }\end{array}$ & No & - & $\begin{array}{c}\text { Yes }(3 \% \text { to } 4 \% \\
\text { PAS + in AL } \\
\text { group) }\end{array}$ & & $\begin{array}{c}\text { Yang et al. } \\
2000\end{array}$ \\
\hline COL:FN mix & Human & Normal & Suspension & - & No & No & No & $\begin{array}{l}\text { KGM (serum } \\
\text { free) }\end{array}$ & No & Bulbus & - & Monolayer culture & $\begin{array}{c}\text { Cook et al. } \\
1998\end{array}$ \\
\hline COL:FN mix & Human & Normal & Suspension & - & No & No & No & EpiLife & No & - & - & & $\begin{array}{c}\text { Gordan } \\
\text { et al. } 2005\end{array}$ \\
\hline COL1 & Bovine & Normal & Suspension & 12 & No & Yes/No & - & DMEM:F12 & $\begin{array}{l}10 \% \\
\text { FBS }\end{array}$ & Bulbus & $\begin{array}{c}\text { Yes (PAS + in } \\
\text { both AL and } \\
\text { submerged } \\
\text { cultures) }\end{array}$ & $\begin{array}{l}\text { Stratified culture } \\
\text { (increased by AL) }\end{array}$ & $\begin{array}{c}\text { Civiale } \\
\text { et al. } 2003\end{array}$ \\
\hline COL1 gel & Rabbit & Normal & Suspension & $7-14$ & No & No & No & DMEM:F12 & $\begin{array}{l}10 \% \\
\text { FBS }\end{array}$ & Bulbus & $\begin{array}{c}\text { Yes (PAS + cell } \\
\text { within gel, PAS- } \\
\text { on the gel surface) }\end{array}$ & $\begin{array}{l}\text { Stratified culture } \\
\text { within gel, } \\
\text { monolayer on the } \\
\text { gel surface }\end{array}$ & $\begin{array}{c}\text { Niiya } \\
\text { et al. } 1997\end{array}$ \\
\hline $\begin{array}{c}\text { COL1 gel } \\
\text { with/without } \\
\text { 3T3 }\end{array}$ & Rabbit & Normal & Suspension & 6 & $\begin{array}{c}3 \mathrm{~T} 3 / \mathrm{N} \\
\mathrm{o}\end{array}$ & Yes/No & No & DMEM:F12 & $\begin{array}{l}10 \% \\
\text { FBS }\end{array}$ & - & $\begin{array}{l}\text { No (PAS-, } \\
\text { MUC5AC-) }\end{array}$ & $\begin{array}{l}\text { Stratified culture } \\
\text { (increased by AL } \\
\text { and } 3 \mathrm{~T} 3 \text { ) }\end{array}$ & $\begin{array}{c}\text { Chen et al. } \\
1994\end{array}$ \\
\hline $\begin{array}{c}\text { COL1 gel } \\
\text { with/without } \\
\text { 3T3 or HCF }\end{array}$ & Human & Normal & Suspension & 14 & $\begin{array}{l}3 \mathrm{~T} 3 / \mathrm{H} \\
\mathrm{CF} / \mathrm{no}\end{array}$ & Yes & No & DMEM:F12 & $\begin{array}{c}5 \% \\
\text { FBS }\end{array}$ & Bulbus & $\begin{array}{c}\text { Yes (only with } \\
\text { HCF) }\end{array}$ & $\begin{array}{l}\text { Stratified culture } \\
\text { (with feeder cells) }\end{array}$ & $\begin{array}{c}\text { Tsai et al. } \\
1994\end{array}$ \\
\hline
\end{tabular}


Table 2. Cont.

\begin{tabular}{|c|c|c|c|c|c|c|c|c|c|c|c|c|c|}
\hline Substrate (s) & $\begin{array}{c}\text { Cell } \\
\text { Species }\end{array}$ & $\begin{array}{c}\text { Normal } \\
\text { Cells/Cells } \\
\text { Line } \\
\end{array}$ & $\begin{array}{c}\text { Explant/ } \\
\text { Suspension } \\
\text { Culture } \\
\end{array}$ & $\begin{array}{c}\text { Culture } \\
\text { Time } \\
\text { (Days) } \\
\end{array}$ & $\begin{array}{l}\text { Feeder } \\
\text { Cells }\end{array}$ & $\begin{array}{c}\text { Air- } \\
\text { Lifting }\end{array}$ & $\begin{array}{c}\text { High } \\
\text { Calcium }\end{array}$ & $\begin{array}{c}\text { Basal } \\
\text { Medium }\end{array}$ & Serum & $\begin{array}{c}\text { Conjunctival } \\
\text { Donor Site }\end{array}$ & Goblet Cells & Comment & Authors \\
\hline $\begin{array}{l}\text { COL1 or } \\
\text { Matrigel }\end{array}$ & Human & $\begin{array}{l}\text { ConjEp- } \\
\text { 1/p53DD/ } \\
\text { cdk4R/ } \\
\text { TERT cell } \\
\text { line }\end{array}$ & Suspension & $\begin{array}{c}\text { (3-7 days } \\
\text { in high } \\
\mathrm{Ca})\end{array}$ & $3 \mathrm{~T} 3 / \mathrm{no}$ & No & Yes & $\begin{array}{l}\text { KM (serum } \\
\text { free) or } \\
\text { DMEM:F12 }\end{array}$ & $\begin{array}{l}10 \% \\
\text { FBS }\end{array}$ & Bulbus & $\begin{array}{l}\text { Yes (MUC5AC } \\
\text { RNA highest with } \\
\text { COL1) }\end{array}$ & $\begin{array}{l}\text { Stratified culture } \\
\text { (COL1), aggregates } \\
\text { (Matrigel) }\end{array}$ & $\begin{array}{c}\text { Gipson } \\
\text { et al. } 2003\end{array}$ \\
\hline $\begin{array}{l}\text { COL1, COL1: } \\
\text { COL3 mix, LN, } \\
\text { FN or Matrigel }\end{array}$ & Rabbit & Normal & Suspension & $<14$ & No & No & No & $\begin{array}{l}\text { PC-1 (serum } \\
\text { free) }\end{array}$ & $\begin{array}{c}0 \text { or } \\
1 \% \\
\text { FBS }\end{array}$ & $\begin{array}{c}\text { All } \\
\text { conjunctiva }\end{array}$ & $\begin{array}{c}\text { Yes ( } 5 \% \text { PAS + in } \\
\text { serum free } \\
\text { cultures on } \\
\text { COL1:COL3 mix) }\end{array}$ & $\begin{array}{l}\text { Stratified culture } \\
\text { (COL1:COL3 mix) }\end{array}$ & $\begin{array}{c}\text { Saha } \\
\text { et al. } 1996\end{array}$ \\
\hline $\begin{array}{l}\text { COL1, Matrigel } \\
\text { or } \\
\text { COL1:Matrigel } \\
\text { mix }\end{array}$ & Rabbit & Normal & Suspension & - & No & No & No & DMEM:F12 & $\begin{array}{l}5 \% \\
\text { FBS }\end{array}$ & $\begin{array}{c}\text { All } \\
\text { conjunctiva }\end{array}$ & $\begin{array}{l}\text { Yes (PAS + cell in } \\
\text { cultures on COL1 } \\
\text { and in globules on } \\
\quad \text { Matrigel) }\end{array}$ & $\begin{array}{c}\text { Monolayer culture } \\
\text { (COL1), aggregates } \\
\text { (Matrigel) }\end{array}$ & $\begin{array}{c}\text { Tsai } \\
\text { et al. } 1988\end{array}$ \\
\hline COL1:FN mix & Rabbit & Normal & Suspension & - & No & No & - & $\begin{array}{l}\text { PC-1 (serum } \\
\text { free) }\end{array}$ & No & - & - & & $\begin{array}{c}\text { Basu } \\
\text { et al. } 1998\end{array}$ \\
\hline COL4 & Rat & Normal & Suspension & 10 & - & Yes & - & $\begin{array}{l}\text { KM (serum } \\
\text { free) or } \\
\text { DMEM:F12 }\end{array}$ & No & Palpebra & - & & $\begin{array}{c}\mathrm{Yu} \\
\text { et al. } 2012\end{array}$ \\
\hline $\begin{array}{c}\text { Compressed } \\
\text { COL }\end{array}$ & Human & Normal & Suspension & 14 & No & No & No & DMEM:F12 & $\begin{array}{l}10 \% \\
\text { FBS }\end{array}$ & - & - & Stratified culture & $\begin{array}{l}\text { Drechsler } \\
\text { et al. } 2015\end{array}$ \\
\hline $\begin{array}{c}\text { Elastin-like } \\
\text { polymer }\end{array}$ & Human & $\begin{array}{l}\text { IOBA- } \\
\text { NHC cell } \\
\text { line }\end{array}$ & Suspension & 5 & No & No & - & DMEM:F12 & - & - & Yes $(\mathrm{Ck} 7+)$ & - & $\begin{array}{c}\text { Martinez- } \\
\text { Osorio } \\
\text { et al. } 2009\end{array}$ \\
\hline
\end{tabular}


Table 2. Cont.

\begin{tabular}{|c|c|c|c|c|c|c|c|c|c|c|c|c|c|}
\hline Substrate (s) & $\begin{array}{c}\text { Cell } \\
\text { Species }\end{array}$ & $\begin{array}{c}\text { Normal } \\
\text { Cells/Cells } \\
\text { Line }\end{array}$ & $\begin{array}{c}\text { Explant/ } \\
\text { Suspension } \\
\text { Culture } \\
\end{array}$ & $\begin{array}{c}\text { Culture } \\
\text { Time } \\
\text { (Days) } \\
\end{array}$ & $\begin{array}{c}\text { Feeder } \\
\text { Cells }\end{array}$ & $\begin{array}{c}\text { Air- } \\
\text { Lifting }\end{array}$ & $\begin{array}{c}\text { High } \\
\text { Calcium }\end{array}$ & $\begin{array}{c}\text { Basal } \\
\text { Medium }\end{array}$ & Serum & $\begin{array}{c}\text { Conjunctival } \\
\text { Donor Site }\end{array}$ & Goblet Cells & Comment & Authors \\
\hline Gelatin-chitosan & Rabbit & Normal & Explant & 14 & No & No & - & DMEM:F12 & $\begin{array}{l}10 \% \\
\text { FBS }\end{array}$ & - & - & $\begin{array}{c}\text { Stratified culture } \\
(\mathrm{Ck} 4+)\end{array}$ & $\begin{array}{c}\text { Zhu } \\
\text { et al. } 2006\end{array}$ \\
\hline $\begin{array}{l}\text { LN-1, LN- } \beta 2 \text { or } \\
\text { COL1 gel with } \\
\text { BCF }\end{array}$ & Bovine & Normal & Explant & 14 & $\begin{array}{c}\mathrm{BCF} / \\
\text { no }\end{array}$ & No & No & $\begin{array}{l}\text { KBM (serum } \\
\text { free) or } \\
\text { DMEM } \\
\text { (serum) }\end{array}$ & $\begin{array}{l}0 \text { or } \\
10 \% \\
\text { FBS }\end{array}$ & Bulbus & - & $\begin{array}{l}\text { Stratified culture } \\
\text { (DMEM/10\% FCS } \\
\text { and cultures on } \\
\text { COL1 with BCF) }\end{array}$ & $\begin{array}{l}\text { Kurpakus } \\
\text { et al. } 1995\end{array}$ \\
\hline $\begin{array}{c}\text { LN-1, LN- } 32 \text { or } \\
\text { poly-I-Iysine }\end{array}$ & Bovine & Normal & Suspension & - & No & No & No & $\begin{array}{l}\text { KBM (serum } \\
\text { free) }\end{array}$ & No & - & - & & $\begin{array}{c}\text { Lin } \\
\text { et al. } 1995\end{array}$ \\
\hline $\mathrm{LN}-10$ & Human & $\begin{array}{l}\text { HC0597 } \\
\text { cell line }\end{array}$ & Suspension & - & No & No & No & $\begin{array}{c}\text { KBM (serum } \\
\text { free) }\end{array}$ & No & - & - & & $\begin{array}{c}\text { Lin } \\
\text { et al. } 2002\end{array}$ \\
\hline
\end{tabular}

$\mathrm{AL}=$ air-lifting; BSA = bovine serum albumin; $\mathrm{COL}=$ collagen; $(-)=$ not reported; DMEM = Dulbecco's Modified Eagle's Medium; PAS = periodic acid-Schiff; $\mathrm{FN}=$ fibronectin; $\mathrm{KGM}=$ keratinocyte growth medium; $\mathrm{FBS}=$ fetal bovine serum; $3 \mathrm{~T} 3=3 \mathrm{~T} 3$ feeder cells; $\mathrm{HCF}=$ human conjunctival fibroblasts; $\mathrm{KM}=$ keratinocyte medium; $\mathrm{LN}=$ laminin; $\mathrm{KDM}=$ keratinocyte basal medium; $\mathrm{FCS}=$ fetal calf serum; $\mathrm{BCF}=$ bovine conjunctival fibroblasts. 


\subsection{Synthetic Polymer Membranes}

Included in this group are polymers of glycolic acid, lactic acid, $\varepsilon$-caprolactone, 1,3-trimethylene carbonate, ethyl acrylate, hydroxyethyl acrylate, and methacrylic acid. One of the benefits of using these polymers is that several of them, including poly(L-lactide-co-glycolide) (PLGA) and poly(e-caprolactone) (PCL), are already approved by the Food and Drug Administration (FDA) for the use in the human body for specific applications. In addition, the biodegradability of these polymers can be adjusted by controlling the ratio and choice of polymers. For instance, PLGA degrades faster than PCL. Furthermore, in contrast to biological membranes, synthetic membranes can be manufactured under sterile conditions, thereby considerably reducing the risk of transferring infectious agents to the patient. Although biodegradable polymers have been investigated at length with various types of cells, only four studies reported biocompatibility with cultured CEC [80-83] (Table 3). Three of these explored growth of CEC on polymer substrates [80-82], whereas one investigated the toxicity of polymer extract on cells cultured on plastic [83]. One of the studies confirmed the presence of MUC5AC positive goblet cells of comparable density to that seen when culturing CEC on AM [80]. The remaining studies did not report presence of goblet cells. The extract study showed lowest to highest viability with 50:50 poly(DL-lactide-co-glycolide) (PDLGA); 85:15 PDLGA and Inion GTR ${ }^{\mathrm{TM}}$, respectively [83]. In cell growth studies, substrates with all three polymers demonstrated high viability [82]. Equally high viability was also seen when growing CEC on poly(ethyl acrylate-co-hydroxyethyl acrylate) (P(EA-co-HEA)) copolymers or 90:10 poly(ethyl acrylate-co-methacrylic acid) (P(EA-co-MAAc)) copolymers [81]. Interestingly, the latter two polymer substrates showed increased adhesion, proliferation and viability when hydrophobicity was increased. In contrast, Ang, et al. demonstrated increased proliferation when decreasing hydrophobicity of their PCL membranes [80]. The latter authors also obtained stratified cultures, which became more stratified by increasing surface hydrophilicity with $\mathrm{NaOH}$. Thus, surface modification of synthetic polymer membranes can affect adhesion, proliferation, viability and stratification. Obvious advantages of synthetic polymer membranes include existing FDA approval for specific uses in the human body, high mechanical strength and biodegradability. 
Table 3. Conjunctival Epithelial cells cultured synthetic polymer membranes.

\begin{tabular}{|c|c|c|c|c|c|c|c|c|c|c|c|c|c|}
\hline Substrate (s) & $\begin{array}{c}\text { Cell } \\
\text { Species }\end{array}$ & $\begin{array}{c}\text { Normal } \\
\text { Cells/Cells } \\
\text { Line } \\
\end{array}$ & $\begin{array}{c}\text { Culture } \\
\text { Time } \\
\text { (Days) } \\
\end{array}$ & $\begin{array}{c}\text { Explant/ } \\
\text { Suspension } \\
\text { Culture } \\
\end{array}$ & $\begin{array}{c}\text { Feeder } \\
\text { Cells }\end{array}$ & $\begin{array}{c}\text { Air- } \\
\text { Lifting }\end{array}$ & $\begin{array}{c}\text { High } \\
\text { Calcium }\end{array}$ & $\begin{array}{l}\text { Culture } \\
\text { Medium }\end{array}$ & Serum & $\begin{array}{c}\text { Conjunctival } \\
\text { Donor Site }\end{array}$ & Goblet Cells & Comment & Authors \\
\hline $\begin{array}{l}\text { 50:50 PDLGA, } \\
\text { 85:15 PDLGA } \\
\text { or Inion GTR }{ }^{\mathrm{TM}}\end{array}$ & Human & $\begin{array}{l}\text { IOBA- } \\
\text { NHC cell } \\
\text { line }\end{array}$ & - & Suspension & No & No & - & DMEM:F12 & $\begin{array}{l}10 \% \\
\text { FBS }\end{array}$ & - & - & $\begin{array}{c}\text { Extract studies } \\
\text { showing lowest to } \\
\text { highest viability } \\
\text { with 50:50 } \\
\text { PDLGA; } 85: 15 \\
\text { PDLGA; Inion } \\
\text { GTR }^{\mathrm{TM}}\end{array}$ & $\begin{array}{l}\text { Huhtala, } \\
\text { et al. } 2008\end{array}$ \\
\hline $\begin{array}{l}\text { 50:50 PDLGA, } \\
\text { 85:15 PDLGA } \\
\text { or Inion GTR }{ }^{\mathrm{TM}}\end{array}$ & Human & $\begin{array}{l}\text { IOBA- } \\
\text { NHC cell } \\
\text { line }\end{array}$ & 3 & Suspension & No & No & - & DMEM:F12 & $\begin{array}{l}10 \% \\
\text { FBS }\end{array}$ & - & - & $\begin{array}{c}\text { High viability with } \\
\text { all types of } \\
\text { polymer }\end{array}$ & $\begin{array}{l}\text { Huhtala, } \\
\text { et al. } 2007\end{array}$ \\
\hline $\begin{array}{c}\text { P(EA-co-HEA) } \\
\text { or 90:10 P(EA- } \\
\text { co-MAAc) } \\
\text { copolymers }\end{array}$ & Human & $\begin{array}{l}\text { IOBA- } \\
\text { NHC cell } \\
\text { line }\end{array}$ & - & Suspension & No & - & - & DMEM:F12 & $\begin{array}{l}10 \% \\
\text { FBS }\end{array}$ & - & - & $\begin{array}{l}\text { All polymers were } \\
\text { non-toxic, } \\
\text { hydrophobicity } \\
\text { increased adhesion, } \\
\text { proliferation and } \\
\text { viability }\end{array}$ & $\begin{array}{l}\text { Campillo- } \\
\text { Fernandez, } \\
\text { et al. } 2007\end{array}$ \\
\hline Ultrathin PCL & Rabbit & Normal & - & $\begin{array}{c}\text { Explant/ } \\
\text { suspension }\end{array}$ & No & No & Yes & $\begin{array}{l}\text { KGM (serum } \\
\text { free) }\end{array}$ & No & - & $\begin{array}{c}\text { Yes (MUC5AC+ } \\
\text { comparable to } \\
\text { AM) }\end{array}$ & $\begin{array}{l}\text { Stratified culture } \\
\text { (increased by } \\
\mathrm{NaOH} \text { ); } \mathrm{NaOH} \\
\text { surface } \\
\text { modification } \\
\text { increased } \\
\text { hydrophilicity and } \\
\text { proliferation }\end{array}$ & $\begin{array}{c}\text { Ang, et al. } \\
2006\end{array}$ \\
\hline
\end{tabular}


Table 3. Cont.

\begin{tabular}{|c|c|c|c|c|c|c|c|c|c|c|c|c|c|}
\hline Substrate (s) & $\begin{array}{c}\text { Cell } \\
\text { Species }\end{array}$ & $\begin{array}{c}\text { Normal } \\
\text { Cells/Cells } \\
\text { Line } \\
\end{array}$ & $\begin{array}{c}\text { Culture } \\
\text { Time } \\
\text { (Days) } \\
\end{array}$ & $\begin{array}{c}\text { Explant/ } \\
\text { Suspension } \\
\text { Culture } \\
\end{array}$ & $\begin{array}{l}\text { Feeder } \\
\text { Cells }\end{array}$ & $\begin{array}{c}\text { Air- } \\
\text { Lifting }\end{array}$ & $\begin{array}{c}\text { High } \\
\text { Calcium }\end{array}$ & $\begin{array}{l}\text { Culture } \\
\text { Medium }\end{array}$ & Serum & $\begin{array}{c}\text { Conjunctival } \\
\text { Donor Site }\end{array}$ & Goblet Cells & Comment & Authors \\
\hline $\begin{array}{l}\text { Temperature- } \\
\text { responsive }\end{array}$ & & & & & & & & & & & & $\begin{array}{l}\text { Stratified culture } \\
\text { ( } 4-5 \text { cell layers); }\end{array}$ & \\
\hline $\begin{array}{l}\text { polymer, } \\
\text { poly(N- } \\
\text { isopropyl- }\end{array}$ & Rabbit & Normal & 10 & Suspension & No & No & No & DMEM:F12 & $\begin{array}{l}10 \% \\
\text { FBS }\end{array}$ & $\begin{array}{l}\text { Fornix/ } \\
\text { palpebra }\end{array}$ & $\begin{array}{c}\text { Yes }(21.5 \% \\
\text { MUC5AC+, } \\
\text { PAS+) }\end{array}$ & $\begin{array}{c}\text { proliferation rate of } \\
38.4 \% \text {; high } \\
\text { viability; Ck4 }\end{array}$ & $\begin{array}{c}\text { Yao, et al. } \\
2015\end{array}$ \\
\hline acrylamide; & & & & & & & & & & & & mRNA increased & \\
\hline PIPAAm) & & & & & & & & & & & & with time & \\
\hline
\end{tabular}

$\mathrm{AL}=$ air-lifting; PDLGA = poly(DL-lactide-co-glycolide); Inion $\mathrm{GTR}^{\mathrm{TM}}=$ a blend of 85:15 poly(L-lactide-co-glycolide) (PLGA) and 70:30 poly(L-lactide-co-1,3trimethylene carbonate) $($ PLTMC) copolymers in a major ratio of 70:30; DMEM = Dulbecco's Modified Eagle's Medium; FBS = fetal bovine serum; $\mathrm{P}($ EA-co-HEA $)=$ poly(ethyl acrylate-co-hydroxyethyl acrylate); $\mathrm{P}(\mathrm{EA}-\mathrm{co}-\mathrm{MAAc})=$ poly $($ ethyl acrylate-co-methacrylic acid $) ; \mathrm{PCL}=$ poly $(\varepsilon$-caprolactone $) ;(-)=$ not reported; MUC5AC = mucin 5AC; PAS = periodic acid-Schiff; $\mathrm{Ck} 4=$ cytokeratin 4 . 


\section{Future Avenues for Developing Tissue Engineered Conjunctival Epithelial Equivalents}

\subsection{Comparative Studies of the Effect of Different Substrates on Cultured Conjunctival Epithelial Cells}

In 2010, Rama and associates described the importance of the phenotype for clinical success following transplantation of cultured limbal epithelial cells [84]. p63, which is a marker for undifferentiated cells, was a significant predictor of clinical outcome [84]. It is possible that the phenotype of cultured CEC will determine success following transplantation of CEC. Comparative studies on how various substrates affect the cell sheet with regard to the phenotype in particular are, therefore, warranted.

\subsection{Storage and Transportation of Cultured Conjunctival Epithelial Cells}

With steadily stricter regulations for cell therapy, which lead to centralization of culture units [85], storage technology of cultured CEC has become increasingly important to allow the tissue to be transported to eye clinics worldwide [86]. Keeping in mind the significance of the phenotype for clinical outcome [84], assessment of the phenotype among other parameters prior to surgery should ideally be performed during the storage period. Moreover, storage in a hermetically sealed container enables microbiological assessment [87]. Finally, storage technology has the advantage of offering increased flexibility in scheduling surgery [88]. Comparative studies on how various substrates influence the ability to store cultured CEC with regard to morphology, viability, and phenotype should be performed to enable worldwide access to cultured CEC.

\section{Conclusion}

Amniotic membrane is the most commonly used substrate for CEC culture. The majority of the studies demonstrated that AM support the growth of goblet cells, in contrast to several alternative substrates. A major weakness in the current literature is the lack of comparative studies, thus such studies should be prioritized to be able to identify the most ideal substrate for ocular surface repair. Considering the disadvantages inherent to the use of a foreign biological material such as AM, clinical studies involving alternative membranes should be carried out as currently only AM has so far been used for transplanting tissue engineered CEC in humans.

\section{Acknowledgments}

Publishing costs were covered by Oslo University Hospital.

\section{Author Contributions}

Jon Roger Eidet, Darlene A. Dartt and Tor Paaske Utheim defined the topic and wrote the review.

\section{Conflicts of Interest}

The authors declare no conflicts of interest. 


\section{References}

1. Wagoner, M.D. Chemical injuries of the eye: Current concepts in pathophysiology and therapy. Surv. Ophthalmol. 1997, 41, 275-313.

2. Gipson, I.K.; Joyce, N.; Zieske, J. The Anatomy and Cell Biology of the Human Cornea, Limbus, Conjunctiva, and Adnexa. In The Cornea; Foster, C.A.D., Dohlman, C., Eds.; Lippincott Williams \& Wilkens: Philadelphia, PA, USA, 2005; pp 1-35.

3. Steven, P.; Gebert, A. Conjunctiva-associated lymphoid tissue-Current knowledge, animal models and experimental prospects. Ophthalmic Res. 2009, 42, 2-8.

4. Schrader, S.; Notara, M.; Beaconsfield, M.; Tuft, S.J.; Daniels, J.T.; Geerling, G. Tissue engineering for conjunctival reconstruction: Established methods and future outlooks. Curr. Eye Res. 2009, 34, 913-924.

5. Yu, D.; Thelin, W.R.; Rogers, T.D.; Stutts, M.J.; Randell, S.H.; Grubb, B.R.; Boucher, R.C. Regional differences in rat conjunctival ion transport activities. Am. J. Physiol. Cell Physiol. 2012, 303, C767-C780.

6. Gendler, S.J.; Spicer, A.P. Epithelial mucin genes. Annu. Rev. Physiol. 1995, 57, 607-634.

7. Fostad, I.G.; Eidet, J.R.; Shatos, M.A.; Utheim, T.P.; Utheim, O.A.; Raeder, S.; Dartt, D.A. Biopsy harvesting site and distance from the explant affect conjunctival epithelial phenotype ex vivo. Exp. Eye Res. 2012, 104, 15-25.

8. Dartt, D.A. Control of mucin production by ocular surface epithelial cells. Exp. Eye Res. 2004, 78, 173-185.

9. Spurr-Michaud, S.; Argueso, P.; Gipson, I. Assay of mucins in human tear fluid. Exp. Eye Res. 2007, 84, 939-950.

10. Argueso, P.; Gipson, I.K. Epithelial mucins of the ocular surface: Structure, biosynthesis and function. Exp. Eye Res. 2001, 73, 281-289.

11. Ramirez-Miranda, A.; Nakatsu, M.N.; Zarei-Ghanavati, S.; Nguyen, C.V.; Deng, S.X. Keratin 13 is a more specific marker of conjunctival epithelium than keratin 19. Mol. Vis. 2011, 17, 1652-1661.

12. Nichols, K.K.; Foulks, G.N.; Bron, A.J.; Glasgow, B.J.; Dogru, M.; Tsubota, K.; Lemp, M.A.; Sullivan, D.A. The international workshop on meibomian gland dysfunction: Executive summary. Invest. Ophthalmol. Vis. Sci. 2011, 52, 1922-1929.

13. Wei, Z.G.; Lin, T.; Sun, T.T.; Lavker, R.M. Clonal analysis of the in vivo differentiation potential of keratinocytes. Invest. Ophthalmol. Vis. Sci. 1997, 38, 753-761.

14. Mason, S.L.; Stewart, R.M.; Kearns, V.R.; Williams, R.L.; Sheridan, C.M. Ocular epithelial transplantation: Current uses and future potential. Regen. Med. 2011, 6, 767-782.

15. Nagasaki, T.; Zhao, J. Uniform distribution of epithelial stem cells in the bulbar conjunctiva. Invest. Ophthalmol. Vis. Sci. 2005, 46, 126-132.

16. Wei, Z.G.; Cotsarelis, G.; Sun, T.T.; Lavker, R.M. Label-retaining cells are preferentially located in fornical epithelium: Implications on conjunctival epithelial homeostasis. Invest. Ophthalmol. Vis. Sci. 1995, 36, 236-246.

17. Lavker, R.M.; Wei, Z.G.; Sun, T.T. Phorbol ester preferentially stimulates mouse fornical conjunctival and limbal epithelial cells to proliferate in vivo. Invest. Ophthalmol. Vis. Sci. 1998, 39, 301-307. 
18. Pe'er, J.; Zajicek, G.; Greifner, H.; Kogan, M. Streaming conjunctiva. Anat. Rec. 1996, 245, 36-40.

19. Chen, W.; Ishikawa, M.; Yamaki, K.; Sakuragi, S. Wistar rat palpebral conjunctiva contains more slow-cycling stem cells that have larger proliferative capacity: Implication for conjunctival epithelial homeostasis. Jpn. J. Ophthalmol. 2003, 47, 119-128.

20. Wei, Z.G.; Wu, R.L.; Lavker, R.M.; Sun, T.T. In vitro growth and differentiation of rabbit bulbar, fornix, and palpebral conjunctival epithelia. Implications on conjunctival epithelial transdifferentiation and stem cells. Invest. Ophthalmol. Vis. Sci. 1993, 34, 1814-1828.

21. Wirtschafter, J.D.; Ketcham, J.M.; Weinstock, R.J.; Tabesh, T.; McLoon, L.K. Mucocutaneous junction as the major source of replacement palpebral conjunctival epithelial cells. Invest. Ophthalmol. Vis. Sci. 1999, 40, 3138-3146.

22. Pellegrini, G.; Golisano, O.; Paterna, P.; Lambiase, A.; Bonini, S.; Rama, P.; de Luca, M. Location and clonal analysis of stem cells and their differentiated progeny in the human ocular surface. J. Cell Biol. 1999, 145, 769-782.

23. Qi, H.; Zheng, X.; Yuan, X.; Pflugfelder, S.C.; Li, D.Q. Potential localization of putative stem/progenitor cells in human bulbar conjunctival epithelium. J. Cell Physiol. 2010, 225, 180-185.

24. Stewart, R.M.; Sheridan, C.M.; Hiscott, P.S.; Czanner, G.; Kaye, S.B. Human conjunctival stem cells are predominantly located in the medial canthal and inferior forniceal areas. Invest. Ophthalmol. Vis. Sci. 2015, 56, 2021-2030.

25. Eidet, J.R.; Fostad, I.G.; Shatos, M.A.; Utheim, T.P.; Utheim, O.A.; Raeder, S.; Dartt, D.A. Effect of biopsy location and size on proliferative capacity of ex vivo expanded conjunctival tissue. Invest. Ophthalmol. Vis. Sci. 2012, 53, 2897-2903.

26. Ramos, T.; Scott, D.; Ahmad, S. An update on ocular surface epithelial stem cells: Cornea and conjunctiva. Stem Cells Int. 2015, 2015, doi:10.1155/2015/601731.

27. Schofield, R. The stem cell system. Biomed. Pharmacother. Biomed. Pharmacother. 1983, 37, 375-380.

28. Watt, F.M.; Hogan, B.L. Out of eden: Stem cells and their niches. Science 2000, 287, 1427-1430.

29. Schlotzer-Schrehardt, U.; Dietrich, T.; Saito, K.; Sorokin, L.; Sasaki, T.; Paulsson, M.; Kruse, F.E. Characterization of extracellular matrix components in the limbal epithelial stem cell compartment. Exp. Eye Res. 2007, 85, 845-860.

30. Radford, C.F.; Rauz, S.; Williams, G.P.; Saw, V.P.; Dart, J.K. Incidence, presenting features, and diagnosis of cicatrising conjunctivitis in the United Kingdom. Eye 2012, 26, 1199-1208.

31. Sobolewska, B.; Deuter, C.; Zierhut, M. Current medical treatment of ocular mucous membrane pemphigoid. Ocul. Surf. 2013, 11, 259-266.

32. Solomon, A.; Espana, E.M.; Tseng, S.C. Amniotic membrane transplantation for reconstruction of the conjunctival fornices. Ophthalmology 2003, 110, 93-100.

33. Patel, B.C.; Sapp, N.A.; Collin, R. Standardized range of conformers and symblepharon rings. Ophthalmic Plast. Reconstr. Surg. 1998, 14, 144-145.

34. Fein, W. Repair of total and subtotal symblepharons. Ophthalmic Surg. 1979, 10, 44-47.

35. Donnenfeld, E.D.; Perry, H.D.; Wallerstein, A.; Caronia, R.M.; Kanellopoulos, A.J.; Sforza, P.D.; D'Aversa, G. Subconjunctival mitomycin C for the treatment of ocular cicatricial pemphigoid. Ophthalmology 1999, 106, 72-79.

36. Thoft, R.A. Conjunctival transplantation. Arch. Ophthalmol. 1977, 95, 1425-1427. 
37. Nakamura, T.; Inatomi, T.; Cooper, L.J.; Rigby, H.; Fullwood, N.J.; Kinoshita, S. Phenotypic investigation of human eyes with transplanted autologous cultivated oral mucosal epithelial sheets for severe ocular surface diseases. Ophthalmology 2007, 114, 1080-1088.

38. Burillon, C.; Huot, L.; Justin, V.; Nataf, S.; Chapuis, F.; Decullier, E.; Damour, O. Cultured autologous oral mucosal epithelial cell sheet (caomecs) transplantation for the treatment of corneal limbal epithelial stem cell deficiency. Invest. Ophthalmol. Vis. Sci. 2012, 53, 1325-1331.

39. Homma, R.; Yoshikawa, H.; Takeno, M.; Kurokawa, M.S.; Masuda, C.; Takada, E.; Tsubota, K.; Ueno, S.; Suzuki, N. Induction of epithelial progenitors in vitro from mouse embryonic stem cells and application for reconstruction of damaged cornea in mice. Invest. Ophthalmol. Vis. Sci. 2004, 45, 4320-4326.

40. Reinshagen, H.; Auw-Haedrich, C.; Sorg, R.V.; Boehringer, D.; Eberwein, P.; Schwartzkopff, J.; Sundmacher, R.; Reinhard, T. Corneal surface reconstruction using adult mesenchymal stem cells in experimental limbal stem cell deficiency in rabbits. Acta Ophthalmol. 2011, 89, 741-748.

41. Gomes, J.A.; Geraldes Monteiro, B.; Melo, G.B.; Smith, R.L.; Cavenaghi Pereira da Silva, M.; Lizier, N.F.; Kerkis, A.; Cerruti, H.; Kerkis, I. Corneal reconstruction with tissue-engineered cell sheets composed of human immature dental pulp stem cells. Invest. Ophthalmol. Vis. Sci. 2010, 51, $1408-1414$.

42. Meyer-Blazejewska, E.A.; Call, M.K.; Yamanaka, O.; Liu, H.; Schlotzer-Schrehardt, U.; Kruse, F.E.; Kao, W.W. From hair to cornea: Toward the therapeutic use of hair follicle-derived stem cells in the treatment of limbal stem cell deficiency. Stem Cells 2011, 29, 57-66.

43. Reza, H.M.; Ng, B.Y.; Gimeno, F.L.; Phan, T.T.; Ang, L.P. Umbilical cord lining stem cells as a novel and promising source for ocular surface regeneration. Stem Cell Rev. 2011, 7, 935-947.

44. Lu, R.; Zhang, X.; Huang, D.; Huang, B.; Gao, N.; Wang, Z.; Ge, J. Conjunctival reconstruction with progenitor cell-derived autologous epidermal sheets in rhesus monkey. PLoS One 2011, 6, doi:10.1371/journal.pone.0025713.

45. Yang, S.P.; Yang, X.Z.; Cao, G.P. Conjunctiva reconstruction by induced differentiation of human amniotic epithelial cells. Genet. Mol. Res. 2015, 14, 13823-13834.

46. Wenkel, H.; Rummelt, V.; Naumann, G.O. Long term results after autologous nasal mucosal transplantation in severe mucus deficiency syndromes. Br. J. Ophthalmol. 2000, 84, 279-284.

47. Tseng, S.C. Amniotic membrane transplantation for ocular surface reconstruction. Biosci. Rep. 2001, 21, 481-489.

48. Touhami, A.; Grueterich, M.; Tseng, S.C. The role of NGF signaling in human limbal epithelium expanded by amniotic membrane culture. Invest. Ophthalmol. Vis. Sci. 2002, 43, 987-994.

49. Lee, S.B.; Li, D.Q.; Tan, D.T.; Meller, D.C.; Tseng, S.C. Suppression of TGF- $\beta$ signaling in both normal conjunctival fibroblasts and pterygial body fibroblasts by amniotic membrane. Curr. Eye Res. 2000, 20, 325-334.

50. Ueta, M.; Kweon, M.N.; Sano, Y.; Sotozono, C.; Yamada, J.; Koizumi, N.; Kiyono, H.; Kinoshita, S. Immunosuppressive properties of human amniotic membrane for mixed lymphocyte reaction. Clin. Exp. Immunol. 2002, 129, 464-470.

51. Solomon, A.; Rosenblatt, M.; Monroy, D.; Ji, Z.; Pflugfelder, S.C.; Tseng, S.C. Suppression of interleukin $1 \alpha$ and interleukin $1 \beta$ in human limbal epithelial cells cultured on the amniotic membrane stromal matrix. Br. J. Ophthalmol. 2001, 85, 444-449. 
52. Kruse, F.E.; Joussen, A.M.; Rohrschneider, K.; You, L.; Sinn, B.; Baumann, J.; Volcker, H.E. Cryopreserved human amniotic membrane for ocular surface reconstruction. Graefe's Arch. Clin. Exp. Ophthalmol. 2000, 238, 68-75.

53. Lee, S.H.; Tseng, S.C. Amniotic membrane transplantation for persistent epithelial defects with ulceration. Am. J. Ophthalmol. 1997, 123, 303-312.

54. Nakamura, T.; Yoshitani, M.; Rigby, H.; Fullwood, N.J.; Ito, W.; Inatomi, T.; Sotozono, C.; Nakamura, T.; Shimizu, Y.; Kinoshita, S. Sterilized, freeze-dried amniotic membrane: A useful substrate for ocular surface reconstruction. Invest. Ophthalmol. Vis. Sci. 2004, 45, 93-99.

55. Russo, A.; Bonci, P.; Bonci, P. The effects of different preservation processes on the total protein and growth factor content in a new biological product developed from human amniotic membrane. Cell. Tissue Banking 2012, 13, 353-361.

56. Von Versen-Hoeynck, F.; Steinfeld, A.P.; Becker, J.; Hermel, M.; Rath, W.; Hesselbarth, U. Sterilization and preservation influence the biophysical properties of human amnion grafts. Biol. J. Int. Assoc. Biol. Standard. 2008, 36, 248-255.

57. Thomasen, H.; Pauklin, M.; Steuhl, K.P.; Meller, D. Comparison of cryopreserved and air-dried human amniotic membrane for ophthalmologic applications. Graefe's Arch. Clin. Exp. Ophthalmol. 2009, 247, 1691-1700.

58. Henderson, H.W.; Collin, J.R. Mucous membrane grafting. Dev. Ophthalmol. 2008, 41, 230-242.

59. Meller, D.; Tseng, S.C. Conjunctival epithelial cell differentiation on amniotic membrane. Invest. Ophthalmol. Vis. Sci. 1999, 40, 878-886.

60. Eidet, J.R.; Utheim, O.A.; Raeder, S.; Dartt, D.A.; Lyberg, T.; Carreras, E.; Huynh, T.T.; Messelt, E.B.; Louch, W.E.; Roald, B.; et al. Effects of serum-free storage on morphology, phenotype, and viability of ex vivo cultured human conjunctival epithelium. Exp. Eye Res. 2012, 94, 109-116.

61. Martinez-Osorio, H.; Calonge, M.; Corell, A.; Reinoso, R.; Lopez, A.; Fernandez, I.; San Jose, E.G.; Diebold, Y. Characterization and short-term culture of cells recovered from human conjunctival epithelium by minimally invasive means. Mol. Vis. 2009, 15, 2185-2195.

62. Fuchs, E.; Green, H. Regulation of terminal differentiation of cultured human keratinocytes by vitamin A. Cell 1981, 25, 617-625.

63. Sangwan, V.S.; Vemuganti, G.K.; Iftekhar, G.; Bansal, A.K.; Rao, G.N. Use of autologous cultured limbal and conjunctival epithelium in a patient with severe bilateral ocular surface disease induced by acid injury: A case report of unique application. Cornea 2003, 22, 478-481.

64. Tan, Y.; Qiu, F.; Qu, Y.L.; Li, C.; Shao, Y.; Xiao, Q.; Liu, Z.; Li, W. Amniotic membrane inhibits squamous metaplasia of human conjunctival epithelium. Am. J. Physiol. Cell Physiol. 2011, 301, C115-C125.

65. Yoshizawa, M.; Feinberg, S.E.; Marcelo, C.L.; Elner, V.M. Ex vivo produced human conjunctiva and oral mucosa equivalents grown in a serum-free culture system. J. Oral Maxillofac. Surg. 2004, 62, 980-988.

66. Tsai, R.J.; Tseng, S.C. Substrate modulation of cultured rabbit conjunctival epithelial cell differentiation and morphology. Invest. Ophthalmol. Vis. Sci. 1988, 29, 1565-1576.

67. Chen, W.Y.; Mui, M.M.; Kao, W.W.; Liu, C.Y.; Tseng, S.C. Conjunctival epithelial cells do not transdifferentiate in organotypic cultures: Expression of K12 keratin is restricted to corneal epithelium. Curr. Eye Res. 1994, 13, 765-778. 
68. Drechsler, C.C.; Kunze, A.; Kureshi, A.; Grobe, G.; Reichl, S.; Geerling, G.; Daniels, J.T.; Schrader, S. Development of a conjunctival tissue substitute on the basis of plastic compressed collagen. J. Tissue Eng. Regen. Med. 2015, doi:10.1002/term.1991.

69. Gordon, Y.J.; Huang, L.C.; Romanowski, E.G.; Yates, K.A.; Proske, R.J.; McDermott, A.M. Human cathelicidin (LL-37), a multifunctional peptide, is expressed by ocular surface epithelia and has potent antibacterial and antiviral activity. Curr. Eye Res. 2005, 30, 385-394.

70. Cook, E.B.; Stahl, J.L.; Miller, S.T.; Gern, J.E.; Sukow, K.A.; Graziano, F.M.; Barney, N.P. Isolation of human conjunctival mast cells and epithelial cells: Tumor necrosis factor-alpha from mast cells affects intercellular adhesion molecule 1 expression on epithelial cells. Invest. Ophthalmol. Vis. Sci. 1998, 39, 336-343.

71. Saha, P.; Kim, K.J.; Lee, V.H. A primary culture model of rabbit conjunctival epithelial cells exhibiting tight barrier properties. Curr. Eye Res. 1996, 15, 1163-1169.

72. Basu, S.K.; Haworth, I.S.; Bolger, M.B.; Lee, V.H. Proton-driven dipeptide uptake in primary cultured rabbit conjunctival epithelial cells. Invest. Ophthalmol. Vis. Sci. 1998, 39, 2365-2373.

73. Kurpakus, M.A.; Lin, L. The lack of extracellular laminin $\beta 2$ chain deposition correlates to the loss of conjunctival epithelial keratin K4 localization in culture. Curr. Eye Res. 1999, 18, 28-38.

74. Lin, L.; Kurpakus Wheater, M. Differential rapid adhesion of bovine ocular surface epithelial cells to laminin isoforms. Curr. Eye Res. 1999, 19, 293-299.

75. Lin, L.; Kurpakus-Wheater, M. Laminin $\alpha 5$ chain adhesion and signaling in conjunctival epithelial cells. Invest. Ophthalmol. Vis. Sci. 2002, 43, 2615-2621.

76. Gipson, I.K.; Spurr-Michaud, S.; Argueso, P.; Tisdale, A.; Ng, T.F.; Russo, C.L. Mucin gene expression in immortalized human corneal-limbal and conjunctival epithelial cell lines. Invest. Ophthalmol. Vis. Sci. 2003, 44, 2496-2506.

77. Zhu, X.; Beuerman, R.W.; Chan-Park, M.B.; Cheng, Z.; Ang, L.P.; Tan, D.T. Enhancement of the mechanical and biological properties of a biomembrane for tissue engineering the ocular surface. Ann. Acad. Med. Singapore 2006, 35, 210-214.

78. Niiya, A.; Matsumoto, Y.; Ishibashi, T.; Matsumoto, K.; Kinoshita, S. Collagen gel-embedding culture of conjunctival epithelial cells. Graefe's Arch. Clin. Exp. Ophthalmol. 1997, 235, 32-40.

79. Martinez-Osorio, H.; Juarez-Campo, M.; Diebold, Y.; Girotti, A.; Alonso, M.; Arias, F.J.; Rodriguez-Cabello, J.C.; Garcia-Vazquez, C.; Calonge, M. Genetically engineered elastin-like polymer as a substratum to culture cells from the ocular surface. Curr. Eye Res. 2009, 34, 48-56.

80. Ang, L.P.; Cheng, Z.Y.; Beuerman, R.W.; Teoh, S.H.; Zhu, X.; Tan, D.T. The development of a serum-free derived bioengineered conjunctival epithelial equivalent using an ultrathin poly(epsilon-caprolactone) membrane substrate. Invest. Ophthalmol. Vis. Sci. 2006, 47, 105-112.

81. Campillo-Fernandez, A.J.; Pastor, S.; Abad-Collado, M.; Bataille, L.; Gomez-Ribelles, J.L.; Meseguer-Duenas, J.M.; Monleon-Pradas, M.; Artola, A.; Alio, J.L.; Ruiz-Moreno, J.M. Future design of a new keratoprosthesis. Physical and biological analysis of polymeric substrates for epithelial cell growth. Biomacromolecules 2007, 8, 2429-2436.

82. Huhtala, A.; Pohjonen, T.; Salminen, L.; Salminen, A.; Kaarniranta, K.; Uusitalo, H. In vitro biocompatibility of degradable biopolymers in cell line cultures from various ocular tissues: Direct contact studies. J. Biomed. Mater. Res. Part A 2007, 83, 407-413. 
83. Huhtala, A.; Pohjonen, T.; Salminen, L.; Salminen, A.; Kaarniranta, K.; Uusitalo, H. In vitro biocompatibility of degradable biopolymers in cell line cultures from various ocular tissues: Extraction studies. J. Mater. Sci. Mater. Med. 2008, 19, 645-649.

84. Rama, P.; Matuska, S.; Paganoni, G.; Spinelli, A.; de Luca, M.; Pellegrini, G. Limbal stem-cell therapy and long-term corneal regeneration. N Engl. J. Med. 2010, 363, 147-155.

85. Daniels, J.T.; Secker, G.A.; Shortt, A.J.; Tuft, S.J.; Seetharaman, S. Stem cell therapy delivery: Treading the regulatory tightrope. Regen. Med. 2006, 1, 715-719.

86. Ahmad, S.; Osei-Bempong, C.; Dana, R.; Jurkunas, U. The culture and transplantation of human limbal stem cells. J. Cell Physiol. 2010, 225, 15-19.

87. Utheim, T.P.; Raeder, S.; Utheim, O.A.; de la Paz, M.; Roald, B.; Lyberg, T. Sterility control and long-term eye-bank storage of cultured human limbal epithelial cells for transplantation. $B r . J$. Ophthalmol. 2009, 93, 980-983.

88. O'Callaghan, A.R.; Daniels, J.T. Concise review: Limbal epithelial stem cell therapy: Controversies and challenges. Stem Cells 2011, 29, 1923-1932.

(C) 2015 by the authors; licensee MDPI, Basel, Switzerland. This article is an open access article distributed under the terms and conditions of the Creative Commons Attribution license (http://creativecommons.org/licenses/by/4.0/). 